\title{
EARLY DIAGNOSTICS OF FUNCTIONAL CHANGES CENTRAL HEMODYNAMICS IN SERONEGATIVE SPONDILOARTHRITIS
}

\author{
Khakimova R.A, Khan T.A. \\ JSC "Republican Specialized Scientific and Practical \\ Medical Center for Therapy and Medical Rehabilitation", \\ Tashkent, Uzbekistan
}

Materials and methods of the study: 20 patients with the diagnosis of ankylosing spondyloratritis (AS) were examined. The diagnosis was verified by the modified New York criteria (1984). Integral rheography (IRGT) was performed in 12 patients with central and in 8 patients with peripheral and rhizomelic forms of the disease. As a control group, 11 healthy individuals were examined. 
Systemic hemodynamics in patients with AS was evaluated by the method of integral body rheography by the method of M.Yu. Tishchenko. Using the obtained parameters, the following parameters characterizing systemic hemodynamics were calculated: the cardiac shock volume indexed to the body surface area (VD), the cardiac index (SI) - the minute volume of the heart indexed to the body surface area; reserve ratio (CR) - the ratio of the actual SR to the proper, determined by the tables of Harris-Benedict, taking into account the growth, body weight, age and sex of the patient; coefficient of respiratory changes (CDI) - the dynamics of blood filling, depending on the phases of the respiratory cycle; an indicator of the intensity of breathing (HDPE) - the product of CDI on the respiratory rate, the coefficient of integral tonicity (CIT) - the relative duration of diastole, reflecting the magnitude of the systemic arterial tone; the tone stabilization index (PTS) is a value characterizing the fluctuations in the systemic arterial tone in different phases of the respiratory cycle.

Results: In patients with AS, there was a tachypnoe. In patients with central lesion $\mathrm{BH}$ was significantly higher than in the second group. CDI, which characterizes the dependence of blood filling of tissues on the phases of the respiratory cycle, was not violated in patients of both groups. HDPE in patients of the first group was significantly increased due to augmentation of $\mathrm{BH}$ (in the second group there were moderate, and in the first group, pronounced changes in the intensity of the functioning of the respiratory system). The parameters of central hemodynamics - HR, UI and SI - in patients of both groups remained within the limits of normal values, as well as $\mathrm{PB}$, characterizing the hydrophilicity of tissues. However, when compared with the data obtained in healthy 
individuals, it is seen that in patients with AS, heart rate and UI significantly increased, which contributes to the increase in SI $(p<0.001$ compared with the indices of healthy individuals). A confirmation of a hyperkinetic type of hemodynamic adjustment is a significant increase in CR, as the ratio of the actual UI to the proper, empirically calculated value. PB in patients with AS was relatively higher than in the control group. CIT indicated a significant increase in the tone of arterioles in patients with AS, while significantly more severe disorders were observed in patients with central lesion. The PST was increased, which corresponded to an increase in vascular tone and a decrease in the vasodilator reserve.

Conclusion: In patients with AS, there are changes in the systemic hemodynamics, leading to the formation of hyperdynamic (irrational) circulatory regime with increased systemic arterial impedance, increased stress of the respiratory system. Changes in the functioning of the respiratory and cardiovascular systems are more pronounced in patients with a central, neuromuscular system - with peripheral forms of the disease. 